POS PROCEEDINGS

\title{
Spin polarization in top pair production in association with two photons at NLO+PS
}

\author{
Gionata Luisoni* \\ Theoretical Physics Department, CERN, Geneva, Switzerland \\ E-mail: gionata.luisoni@cern.ch
}

\begin{abstract}
This talk focuses on the impact of top-quark spin polarization effects in Higgs boson production in association with a top-quark pair, where the Higgs boson decays to two photons. Predictions for the signal are compared with direct top-quark pair production in association with two photons at NLO+PS.
\end{abstract}

XXV International Workshop on Deep-Inelastic Scattering and Related Subjects 3-7 April 2017

University of Birmingham, $U K$

\footnotetext{
* Speaker.
} 


\section{Introduction}

In the last decade the development of automated tools for precise calculations of high energy scattering processes has undergone a dramatic acceleration. Nowadays many tools exist which allow to automatically or semi-automatically compute Next-to-Leading-Order (NLO) radiative corrections in perturbation theory both in Quantum Chromodynamics (QCD) and in the Electroweak (EW) sector of the Stander Model (SM). Interfacing the various tools to each other allows for cross checks and increases their flexibility and their versatility. This is also the purpose of the interface developed in [1] between the One Loop Provider (OLP) GOSAM [2] and the multipurpose NLO Monte Carlo tool MADGRAPH5_AMC@NLO [3], which we shall review here. As a first application the two codes were used jointly to compute spin polarization observables in $t \bar{t} \gamma \gamma$ production.

\section{GOSAM-MADGRAPH5_AMC@NLO interface and validation}

The GoSAM package allows to generate and evaluate one-loop QCD and EW corrections and on top of the GoSAM code itself, consists of several programs: QGRAF [4], FORM [5] and SPINNEY [6] for the generation and computation of the algebraic expressions, NINJA [7], SAMURAi [8] and GolEm95C [9] for the reduction of the 1-loop amplitudes and QCDLoop [10] or ONELOOP [11] for the evaluation of the scalar loop integrals. NinJA implements an integrand reduction technique based on the Laurent expansion [12], SAMURAI uses the OPP reduction method [13] in $d$ dimensions, whereas GoLEM95C is a tensor integral library. For the latter the reconstruction of the tensor structure is performed either using the method outlined in [14] or via derivation with respect to the loop momentum. MADGRAPH5_AMC@NLO is instead a framework for the computation of differential cross sections at the LO, NLO and NLO matched to Parton Shower (PS) level. The subtraction of the infrared divergences at NLO is performed using the FKS method [15] and for the computation of the virtual amplitude it can rely on an internal code called MADLOOP [16] or on external OLPs like GoSAM. The matching of NLO and PS is performed in the MC@NLO scheme [17], available for several PS codes. More about the its internal algorithms can be found in Ref. [18].

The interface between GoSAM and MADGRAPH5_AMC@NLO is based on the standards defined in the first BLHA interface [19]. When running the MADGRAPH5_AMC@NLO interactive session, the command

\section{\$ set OLP GoSam}

changes the employed OLP from its default MADLOOP to GoSAM. All the basic information about a given process, as well as the full list of partonic subprocesses that need to be computed by GoSAM are communicated between the MC and the OLP via the BLHA order and contract file system. For static parameters, which do not change at each phase space point, but stay constant during the MC integration and event generation, the information is passed via a SUSY Les Houches Accord (SLHA) parameter file. This is created by MADGRAPH5_AMC@NLO and read by GoSAM. The generation of the one loop amplitudes can be further customized by editing by hand a separate input file for GoSAM. After the input file is ready, any NLO process can be generated following 
the general MADGRAPH5_AMC@NLO procedure. More details about the interface can be found in [1].

The interface was validated performing several checks and comparison at the single phase space point level between GOSAM and MADLOOP, and at the level of total cross section for a number of different processes. Comparison plots and tables are shown in [1].

\section{Spin polarization results}

In this section we present results at NLO+PS level for the LHC at $13 \mathrm{TeV}$ and compare the background process $t \bar{t} \gamma \gamma$, where the photons are directly radiated from the quarks, with the signal process $t \bar{t} H$ in which the Higgs boson decays to two photons. The study is performed using NLO predictions for $t \bar{t} H$ and the continuum $t \bar{t} \gamma \gamma$ production. The top and anti-top quarks are subsequently decayed semi-leptonically $t \rightarrow W^{+}\left(\rightarrow \bar{l} v_{l}\right) b, \bar{t} \rightarrow W^{-}\left(\rightarrow l, \bar{v}_{l}\right) \bar{b}$ with MADSPIN [20], taking into account spin correlation effects, and then showered and hadronised with PYTHIA 8.2 [21], using its default parameters, but with the underlying event turned off. The short-distance events were generated and compared with two slightly different sets of cuts in order to verify that they had no impact on the results at the level of the analysis. Note that for the background process we neglect effects of photon bremsstrahlung from the charged top decay products, which can at least partially be reduced by applying proper kinematical cuts. A similar analysis was carried out at LO in Ref. [22]. The analysis cuts are designed to increase the signal over the background, but are by no means optimised to maximise the enhancement. The two photons from the Higgs decay (or the two hard photons in the $t \bar{t} \gamma \gamma$ process) are required to be isolated and fulfill

$$
p_{T, \gamma}>20 \mathrm{GeV}, \quad\left|\eta_{\gamma}\right|<2.5, \quad 123 \mathrm{GeV}<m_{\gamma \gamma}<129 \mathrm{GeV}
$$

where the invariant mass requirement selects a window around the Higgs boson mass, which reduces the background significantly without altering the signal strength. Furthermore, we require the events to have two oppositely charged leptons and two b-jets coming from the top and anti-top decays. The leptons are selected requiring

$$
p_{T, l^{ \pm}}>10 \mathrm{GeV}, \quad\left|\eta_{l^{ \pm}}\right|<2.7 .
$$

The b-jets are defined to be jets containing at least one lowest lying B meson. The jets themselves are defined by clustering all stable hadrons and photons, but excluding the two photons selected using Eq. (3.1), using the anti- $k_{T}$ algortithm as implemented in the code FASTJET [23, 24], with

$$
\Delta R=0.4, \quad p_{T, j}>20 \mathrm{GeV}, \quad\left|\eta_{j}\right|<4.7 .
$$

We use MC truth information to select the photons from the Higgs decay (signal) or hard events (background) as well as the leptons and b-jets coming from the top and anti-top decays. As reconstructed (anti-)top quark, we use the four-momentum of the (anti-)top quark just before it decays, as provided in the Pythia 8 event record.

We now focus on observables that allow for investigating polarisation effects of the top and anti-top quarks as well as of their decay products. This can be done by studying angular variables which involve the decay products of the top and anti-top quarks. Typically, for hadronic 

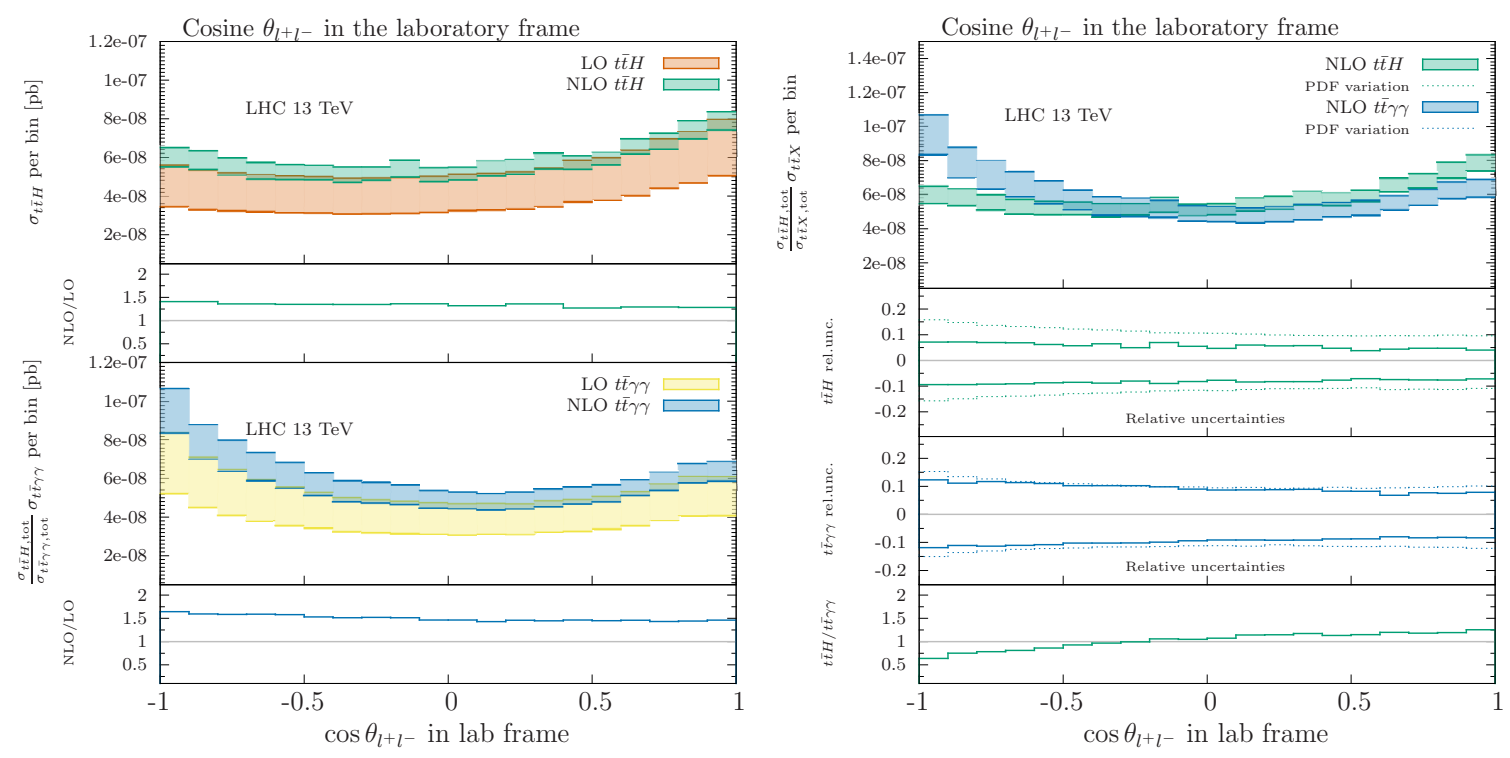

Figure 1: $\cos \theta_{l l}$ distribution for the signal $(t \bar{t} H)$ and background $(t \bar{t} \gamma \gamma)$ processes in the laboratory frame. The exact definition of the angle $\theta$ is given in the text. The $t \bar{t} \gamma \gamma$ prediction is normalized to the $t \bar{t} H$ inclusive cross-section. On the left hand side, we compare LO with NLO predictions and show their K-factor separately for $t \bar{t} H$ and $t \bar{t} \gamma \gamma$ in bottom insets. On the right hand side, we show NLO relative uncertainties with the signal-to-background ratio as the last bottom inset.

$t \bar{t}$-production, very specific kinematic frames are defined [25]. In the following we will consider the three-dimensional opening angle $\theta_{l l}$ between the leptonic decay products of the top $\left(l^{+}\right)$and anti-top quarks $\left(l^{-}\right)$, defined in three different frames. The most straightforward possibility is to define $\theta_{l l}$ in the laboratory frame (referred to as lab-frame in the following). For the remaining two frames we define $\theta_{l l}$ to be the angle between the direction of flight of $l^{+}$, measured in frame where the top quark is at rest, and the direction of flight of $l^{-}$, measured in the frame where the anti-top quark is at rest. Since two rest frames are involved in this definition, a common initial frame needs to be specified, from which the (rotation-free) Lorentz boost can be applied in order to transform the system to the $t$ and $\bar{t}$ rest frames. We choose two possible starting points, which we label as frame- 1 and frame-2, defined as follows:

- frame-1: the Lorentz boosts to bring $t$ and $\bar{t}$ separately at rest are defined with respect to the $t \bar{t}$-pair center-of-mass frame,

- frame-2: the Lorentz boosts to bring $t$ and $\bar{t}$ separately at rest are defined with respect to the lab-frame.

In Figure 1 we show the behaviour of $\cos \theta_{l l}$ in the lab-frame. To highlight shape differences, the background predictions have been normalized to the inclusive $t \bar{t} H$ cross section. The left hand side of Figure 1 presents the comparison of the LO and NLO predictions for $t \bar{t} H$ and $t \bar{t} \gamma \gamma$ separately and shows their respective differential K-factors. The right hand side histograms compare results for the signal and background processes, with their ratio and respective relative uncertainty in the bottom insets. This is to be compared with the plots in Figure 2, where the same observable is 

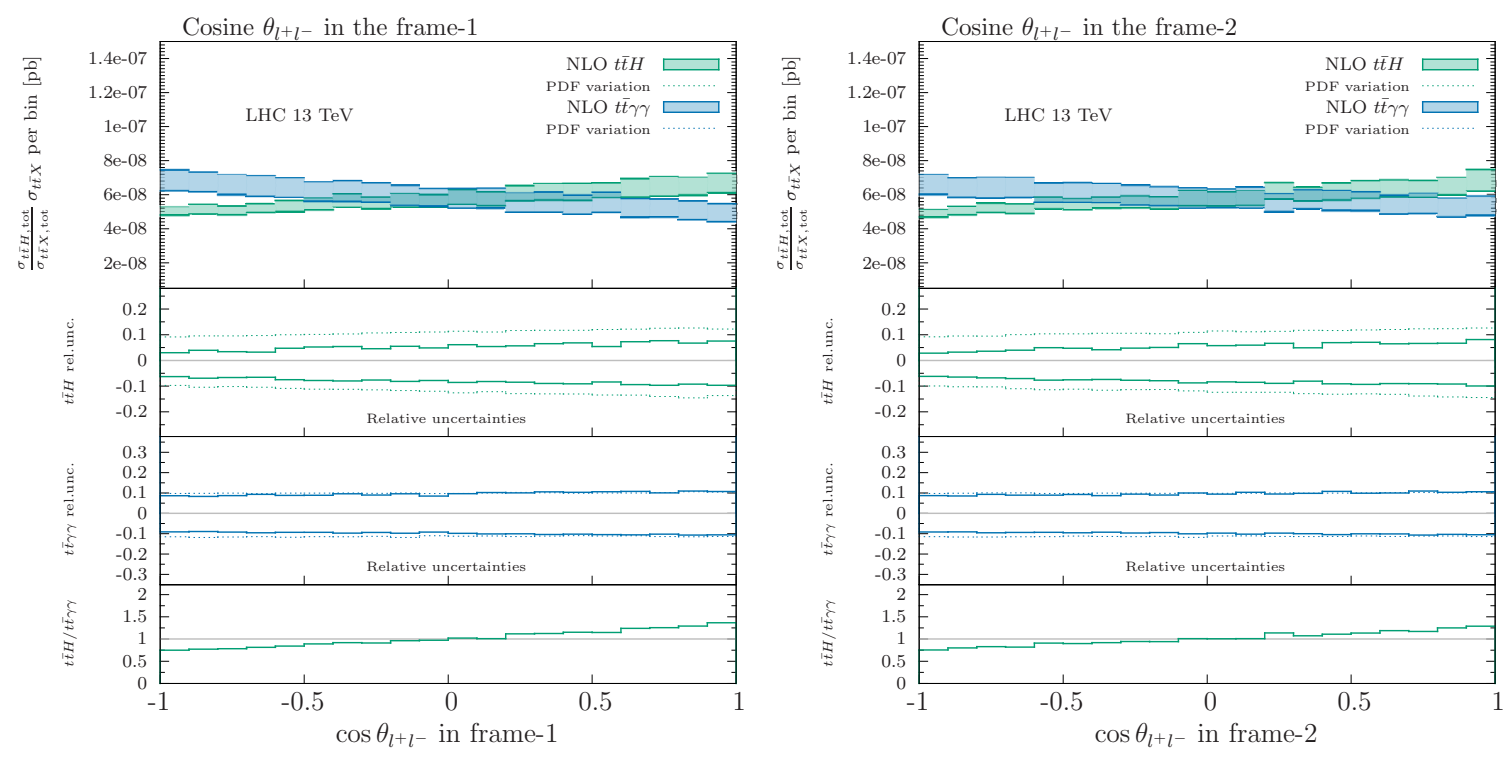

Figure 2: Signal-background comparison as shown on the right of Figure 1, but for reference frame-1 (left) and reference frame-2 (right).

shown in frame- 1 and frame- 2 for signal and background. In the two latter frames a difference in the sign of the slope emerges, while in the lab frame, despite a clear difference in the slopes, the curves have an analogous trend. By comparing the two ratio plots at the bottom in Figure 2, we conclude that the frame-1 offers the best signal-to-background ratio. Although not shown here, it is also worth stressing that, while in the lab frame the K-factors tend to decrease slightly when $\cos \theta_{l l} \rightarrow$ 1 , in frame-1 and frame-2, the NLO corrections feature an almost perfectly flat K-factor. Many more details about this analysis and several further comparisons between signal and background predictions can be found in original publication [1].

\section{References}

[1] H. van Deurzen, R. Frederix, V. Hirschi, G. Luisoni, P. Mastrolia and G. Ossola, Eur. Phys. J. C 76 (2016) no.4, 221 [arXiv:1509.02077].

[2] G. Cullen, N. Greiner, G. Heinrich, G. Luisoni, P. Mastrolia, G. Ossola, T. Reiter and F. Tramontano, Eur. Phys. J. C 72 (2012) 1889 [arXiv:1111.2034]; G. Cullen et al., Eur. Phys. J. C 74 (2014) no.8, 3001 [arXiv:1404.7096].

[3] J. Alwall et al., JHEP 1407 (2014) 079 [arXiv:1405.0301].

[4] P. Nogueira, J. Comput. Phys. 105 (1993) 279.

[5] J. Kuipers, T. Ueda, J. A. M. Vermaseren and J. Vollinga, Comput. Phys. Commun. 184 (2013) 1453 [arXiv:1203.6543].

[6] G. Cullen, M. Koch-Janusz and T. Reiter, Comput. Phys. Commun. 182 (2011) 2368 [arXiv:1008.0803].

[7] T. Peraro, Comput. Phys. Commun. 185 (2014) 2771 [arXiv:1403.1229].

[8] P. Mastrolia, G. Ossola, T. Reiter and F. Tramontano, JHEP 1008 (2010) 080 [arXiv:1006.0710]. 
[9] T. Binoth, J.-P. Guillet, G. Heinrich, E. Pilon and T. Reiter, Comput. Phys. Commun. 180 (2009) 2317 [arXiv:0810.0992]; G. Cullen, J. P. Guillet, G. Heinrich, T. Kleinschmidt, E. Pilon, T. Reiter and M. Rodgers, Comput. Phys. Commun. 182 (2011) 2276 [arXiv:1101.5595]; J. P. Guillet, G. Heinrich and J. F. von Soden-Fraunhofen, Comput. Phys. Commun. 185 (2014) 1828 [arXiv:1312.3887].

[10] G. J. van Oldenborgh, Comput. Phys. Commun. 66 (1991) 1. R. K. Ellis and G. Zanderighi, JHEP 0802 (2008) 002 [arXiv:0712.1851]; S. Carrazza, R. K. Ellis and G. Zanderighi, Comput. Phys. Commun. 209 (2016) 134 [arXiv:1605.03181].

[11] A. van Hameren, Comput. Phys. Commun. 182 (2011) 2427 [arXiv:1007.4716].

[12] P. Mastrolia, E. Mirabella and T. Peraro, JHEP 1206 (2012) 095 Erratum: [JHEP 1211 (2012) 128] [arXiv:1203.0291]; H. van Deurzen, G. Luisoni, P. Mastrolia, E. Mirabella, G. Ossola and T. Peraro, JHEP 1403 (2014) 115 [arXiv:1312.6678];

[13] G. Ossola, C. G. Papadopoulos and R. Pittau, Nucl. Phys. B 763 (2007) 147 [hep-ph/0609007]. G. Ossola, C. G. Papadopoulos and R. Pittau, JHEP 0805 (2008) 004 [arXiv:0802.1876]. P. Mastrolia, G. Ossola, C. G. Papadopoulos and R. Pittau, JHEP 0806 (2008) 030 [arXiv:0803.3964].

[14] G. Heinrich, G. Ossola, T. Reiter and F. Tramontano, JHEP 1010 (2010) 105 [arXiv:1008.2441].

[15] S. Frixione, Z. Kunszt and A. Signer, Nucl. Phys. B 467 (1996) 399 [hep-ph/9512328]; R. Frederix, S. Frixione, F. Maltoni and T. Stelzer, JHEP 0910 (2009) 003 [arXiv:0908.4272].

[16] V. Hirschi, R. Frederix, S. Frixione, M. V. Garzelli, F. Maltoni and R. Pittau, JHEP 1105 (2011) 044 [arXiv:1103.0621].

[17] S. Frixione and B. R. Webber, JHEP 0206 (2002) 029 [hep-ph/0204244].

[18] J. Alwall, M. Herquet, F. Maltoni, O. Mattelaer and T. Stelzer, JHEP 1106 (2011) 128 [arXiv:1106.0522].

[19] T. Binoth et al., Comput. Phys. Commun. 181 (2010) 1612 [arXiv:1001.1307]. S. Alioli et al., Comput. Phys. Commun. 185 (2014) 560 [arXiv:1308.3462].

[20] S. Frixione, E. Laenen, P. Motylinski and B. R. Webber, JHEP 0704 (2007) 081 [hep-ph/0702198]; P. Artoisenet, R. Frederix, O. Mattelaer and R. Rietkerk, JHEP 1303 (2013) 015 [arXiv:1212.3460].

[21] T. Sjöstrand et al., Comput. Phys. Commun. 191 (2015) 159 [arXiv:1410.3012].

[22] S. Biswas, R. Frederix, E. Gabrielli and B. Mele, JHEP 1407 (2014) 020 [arXiv:1403.1790].

[23] M. Cacciari and G. P. Salam, Phys. Lett. B 641 (2006) 57 [hep-ph/0512210]; M. Cacciari, G. P. Salam and G. Soyez, JHEP 0804 (2008) 063 [arXiv:0802.1189].

[24] M. Cacciari, G. P. Salam and G. Soyez, Eur. Phys. J. C 72 (2012) 1896 [arXiv:1111.6097].

[25] W. Bernreuther, A. Brandenburg, Z. G. Si and P. Uwer, Phys. Rev. Lett. 87 (2001) 242002 [hep-ph/0107086]; W. Bernreuther, A. Brandenburg, Z. G. Si and P. Uwer, Nucl. Phys. B 690 (2004) 81 [hep-ph/0403035]; W. Bernreuther and Z. G. Si, Nucl. Phys. B 837 (2010) 90 [arXiv:1003.3926]. 